\title{
Correction: Educational delay and attainment in persons with neurofibromatosis 1 in Denmark
}

\author{
Karoline Doser ${ }^{1} \cdot$ Line Kenborg ${ }^{2}$ - Elisabeth Wreford Andersen ${ }^{1} \cdot$ Pernille Envold Bidstrup ${ }^{1}$ - Anja Kroyer ${ }^{2}$. \\ Hanne Hove ${ }^{3}$. John Østergaard ${ }^{4}$. Sven Asger Sørensen ${ }^{5}$. Christoffer Johansen ${ }^{1,6}$ • John Mulvihill ${ }^{7}$. \\ Jeanette Falck Winther ${ }^{2,8} \cdot$ Susanne Oksbjerg Dalton ${ }^{1}$
}

Published online: 22 March 2019

(c) European Society of Human Genetics 2019

\section{Correction to: Eur J Hum Genet \\ https://doi.org/10.1038/s41431-019-0359-8 \\ published online 28 Feb 2019}

Since the publication of the article, the authors noticed that 'NFI cohort' and 'NFI-free cohort' columns in the
'Autism' ${ }^{\mathrm{g}}$ ' and the 'Autism/ADHD' rows had been erroneously interchanged in Table 3. The updated table can be found below. This has now been updated in the HTML and PDF of the original article.

These authors contributed equally: Jeanette Falck Winther, Susanne Oksbjerg Dalton

The original article can be found online at https://doi.org/10.1038/ s41431-019-0359-8.

Karoline Doser

kdoser@cancer.dk

1 Survivorship Unit, Danish Cancer Society Research Center, Copenhagen, Denmark

2 Childhood Cancer Research Group, Danish Cancer Society Research Center, Copenhagen, Denmark

3 The RAREDIS Database, Centre for Rare Diseases, Copenhagen University Hospital and Aarhus University, Aarhus, Denmark

4 Center for Rare Disease, Aarhus University Hospital, Aarhus, Denmark

5 Copenhagen University, Copenhagen, Denmark

6 Oncology Clinic, Finsen Center, Rigshospitalet, 5073 Copenhagen, Denmark

7 University of Oklahoma Health Sciences Center, Oklahoma City, OK, USA

8 Department of Clinical Medicine, Faculty of Health, Aarhus University, Aarhus, Denmark 
Table 3 Descriptive characteristics of the 1065 Danish persons with NF1 and the 8791 NF1-free persons, born 1965-2000, graduated from grade 9 in Denmark 1981-2015

\begin{tabular}{|c|c|c|c|c|c|c|c|c|}
\hline & \multicolumn{4}{|c|}{ NF1 cohort } & \multicolumn{4}{|c|}{ NF1-free cohort } \\
\hline & $N$ & $\%$ & Grad. & Person-years & $N$ & $\%$ & Grad. & Person-years \\
\hline Total & 1,065 & & 932 & 2,734 & 8,791 & & 7,962 & 19,274 \\
\hline \multicolumn{9}{|l|}{ Gender } \\
\hline Male & 529 & 50 & 454 & 1,416 & 4,426 & 50 & 3,897 & 10,216 \\
\hline Female & 536 & 50 & 478 & 1,318 & 4,365 & 50 & 4,065 & 9,058 \\
\hline \multicolumn{9}{|c|}{ Pearson $\chi^{2}(1)=0.17$ Pr. $=0.68$} \\
\hline \multicolumn{9}{|l|}{ Birth year } \\
\hline 1965-1969 & 107 & 10 & 102 & 260 & 960 & 11 & 916 & 2,187 \\
\hline 1970-1974 & 151 & 14 & 144 & 341 & 1,248 & 14 & 1,208 & 2,687 \\
\hline $1975-1979$ & 136 & 13 & 128 & 351 & 1,025 & 12 & 996 & 2,278 \\
\hline $1980-1984$ & 133 & 13 & 119 & 412 & 907 & 10 & 869 & 2,082 \\
\hline 1985-1989 & 128 & 12 & 115 & 384 & 934 & 10 & 890 & 2,249 \\
\hline 1990-1994 & 164 & 15 & 153 & 465 & 1,375 & 16 & 1,314 & 3,330 \\
\hline 1995-2002 & 246 & 23 & 171 & 520 & 2,342 & 27 & 1,769 & 4,461 \\
\hline \multicolumn{9}{|c|}{ Pearson $\chi^{2}(6)=12.20 \operatorname{Pr}=0.058$} \\
\hline \multicolumn{9}{|c|}{ Mother's education } \\
\hline Short & 343 & 32 & 298 & 891 & 2,234 & 25 & 2,003 & 5,381 \\
\hline Medium & 502 & 47 & 439 & 1,317 & 4,273 & 49 & 3,914 & 9,199 \\
\hline Long & 189 & 18 & 168 & 434 & 2,097 & 24 & 1,896 & 4,211 \\
\hline Missing & 31 & 3 & & & 187 & 2 & & \\
\hline \multicolumn{9}{|c|}{ Pearson $\chi^{2}(3)=35.11 \operatorname{Pr}=0.000$} \\
\hline \multicolumn{9}{|l|}{ Cancer $^{\mathrm{a}}$} \\
\hline Yes & 57 & 5 & 50 & 148 & 49 & 1 & 48 & 102 \\
\hline \multicolumn{9}{|c|}{ Pearson $\chi^{2}(1)=205.26 \operatorname{Pr}=0.000$} \\
\hline \multicolumn{9}{|c|}{ Diseases of bone, joints and soft tissue ${ }^{b}$} \\
\hline & 76 & 7 & 70 & 192 & 137 & 2 & 126 & 306 \\
\hline \multicolumn{9}{|c|}{ Pearson $\chi^{2}(1)=139.77 \operatorname{Pr}=0.000$} \\
\hline \multicolumn{9}{|c|}{ Diseases of nervous system ${ }^{c}$} \\
\hline & 155 & 15 & 126 & 479 & 468 & 5 & 406 & 1,136 \\
\hline \multicolumn{9}{|c|}{ Pearson $\chi^{2}(1)=136.68 \operatorname{Pr}=0.000$} \\
\hline \multicolumn{9}{|c|}{ Diseases of circulatory system ${ }^{\mathrm{d}}$} \\
\hline & 25 & 2 & 19 & 62 & 34 & 0 & 32 & 72 \\
\hline \multicolumn{9}{|c|}{ Pearson $\chi^{2}(1)=61.37 \operatorname{Pr}=0.000$} \\
\hline \multicolumn{9}{|c|}{ Other medical diseases ${ }^{\mathrm{e}}$} \\
\hline & 533 & 50 & 461 & 1,442 & 3,071 & 35 & 2,746 & 6,938 \\
\hline \multicolumn{9}{|c|}{ Pearson $\chi^{2}(1)=93.54 \operatorname{Pr}=0.000$} \\
\hline \multicolumn{9}{|l|}{ Anxiety ${ }^{\mathrm{f}}$} \\
\hline & 7 & 1 & 5 & 17 & 48 & 1 & 42 & 123 \\
\hline Pearson $\chi^{2}(1)$ & $=0.645$ & & & & & & & \\
\hline Autism $^{\mathrm{g}}$ & & & & & & & & \\
\hline & 23 & 2 & 15 & 92 & 92 & 1 & 75 & 295 \\
\hline Pearson $\chi^{2}(1)$ & $r=0.00$ & & & & & & & \\
\hline $\mathrm{ADHD}^{\mathrm{h}}$ & & & & & & & & \\
\hline & 50 & 5 & 39 & 114 & 114 & 1 & 94 & 324 \\
\hline Pearson $\chi^{2}(1)$ & $r=0.00$ & & & & & & & \\
\hline Autism/ADHD & & & & & & & & \\
\hline & 69 & 7 & 50 & 198 & 199 & 2 & 163 & 601 \\
\hline Pearson $\chi^{2}(1)$ & $r=0.00$ & & & & & & & \\
\hline Depression anc & chiatric & & & & & & & \\
\hline & 172 & 16 & 128 & 499 & 497 & 6 & 405 & 1,432 \\
\hline Pearson $\chi^{2}(1)$ & $\operatorname{Pr}=0.0$ & & & & & & & \\
\hline
\end{tabular}


Table 3 (continued)

\begin{tabular}{|c|c|c|c|c|c|c|c|c|}
\hline & \multicolumn{4}{|c|}{ NF1 cohort } & \multicolumn{4}{|c|}{ NF1-free cohort } \\
\hline & $N$ & $\%$ & Grad. & Person-years & $N$ & $\%$ & Grad. & Person-years \\
\hline \multicolumn{9}{|c|}{ Age at graduation (years) } \\
\hline $14-15$ & & & 10 & 1,047 & & & 46 & 8,622 \\
\hline $15-15.5$ & & & 44 & 501 & & & 579 & 4,090 \\
\hline $15.5-16$ & & & 278 & 413 & & & 3,189 & 3,074 \\
\hline $16-16.5$ & & & 348 & 235 & & & 3,250 & 1,260 \\
\hline $16.5-17$ & & & 170 & 110 & & & 703 & 345 \\
\hline $17-18$ & & & 77 & 83 & & & 186 & 315 \\
\hline $18-20$ & & & 5 & 346 & & & 9 & 1,568 \\
\hline
\end{tabular}

a Cancer; malignant (ICD-10): C00-C43, C46-C96, B21.0, D30.1-D30.9, D32-D33, D35.2-D35.4, D41.4, D42-D43, D44.3-D44.5, benign (ICD-8): 210-221, 223.0, 224, 225.5-225.6, 226.0-226.1, 226.4-228

${ }^{\mathrm{b}}$ Diseases of bone, joints soft tissue (ICD-8): 720-738, 274, 710-718

${ }^{c}$ Diseases of nervous system (ICD-8): 290, 320-324, 340-342, 344-347, 349-358, 360-389

${ }^{\mathrm{d}}$ Diseases of circulatory system (ICD-8): 390-398, 400-404, 410-414, 420-438, 440-448, 450-458

${ }^{\mathrm{e}}$ Other medical diseases (ICD-8): 000-136, 240-242, 244-269, 275.4-276, 278-279, 606, 626-628, 280-281, 283-289, 460-519, 520-543, 550$577,580-605,607-625,629,700-709,680-686,690-698,274,710-718,720-738$

${ }^{\mathrm{f}}$ Anxiety (ICD-10): F41, Depression (ICD-10): F32

${ }^{\mathrm{g}}$ Autism (ICD-10): F84

${ }^{\mathrm{h}} \mathrm{ADHD}$ (ICD-10): F90

iDepression and other psychiatric disease (ICD-10): F01-99, excluding F41, F84, F90 